global hydrological cycle. Geophys. Res. Lett., 31, doi: 10.1029/2003GL019188.

Wild, M., H. Gilgen, A. Rösch, A. Ohmura, C. N. Long, E. G. Dutton, B. Forgan, A. Kallis, V. Russak, and
A. Tsvetkov, 2005: From dimming to brightening: Decadal Changes in Solar Radiation at Earth's Surface, Science, 308, 847-850.

\title{
Mineral dust records from Greenland ice cores
}

\section{Urs Ruth}

\section{Alfred-Wegener-Institut für Polar- und Meeresforschung, Bremerhaven, Germany; uruth@awi-bremerhaven.de}

Mineral dust plays an important role in climate research today. Through its radiative properties, mineral dust aerosol directly influences the radiative balance of the atmosphere. Furthermore, mineral dust is believed to be climatically active in a number of indirect ways. These include its ability to act as cloud-condensation nuclei with consequences for the amount and characteristics of cloud cover, as well as its potential to control primary bioproductivity in remote ocean and land areas that would otherwise be nutrient limited (fertilisation). Although not all of these processes are yet well understood the great dynamics of the mineral dust cycle in the past allows for an important feedback loop for climatic changes. On this question it is not yet certain whether the great concentration increase during glacial times, as observed in polar ice cores, is restricted to the high latitudes or whether it is representative for larger parts of the atmosphere.

Terrestrial, marine and ice-core archives of windblown mineral dust can be used to infer past climate conditions relevant to the dust cycle. For this purpose, it is very fortunate that - at least for Greenlandic ice cores-the source regions of mineral dust have been very well localised: The East Asian deserts in Western China and Inner Mongolia have been identified through isotopic and mineralogical studies as predominant sources for recent times as well as for the last glacial period (Fig. 1) (Svensson et al., 2000, Bory et al., 2003). Therefore, dust from ice cores is distinct from most other proxy parameters for which the sources are neither well known nor localised in space. Accordingly, an idealised concept for interpreting Greenlandic ice core dust records consists of (i) mobilisation of dust in the source and uplift into the free troposphere, (ii) long-range transport with the westerly jets over a source-free area, (iii) loss processes en route due to gravitational settling and wash-out, and (iv) deposition onto the ice sheet. The total mineral dust load is divided into a soluble fraction, which is best approximated by $\mathrm{Ca}^{2+}$ ion concentrations, and an insoluble fraction, which for practical reasons is most commonly inferred from the volume of water-insoluble particles in molten ice-core samples. Usually, both fractions vary alike and point to only small though significant compositional variations. Apart from the concentration, the size distribution of insoluble dust particles can also be measured, which provides unique information on changes of past atmospheric long-range transport.

Continuous time series of insoluble dust particle concentration and size distribution from the North-GRIP ice core are shown in Figure 2 (Ruth et al., 2003). The concentration exhibits a strong interglacial-glacial increase by a factor of $\sim 100$ and an interstadial-stadial increase by a factor of typically $\sim 10$. The changes are very rapid and therefore point to atmospheric processes as their main drivers. The two most likely mechanisms to produce high ice core dust levels are (a) increased mobilisation in the source area due to higher wind speeds or more frequent dust storms, and (b) increased long-range transport efficiency due to shorter transit times or less wash-out en route. It is a matter of debate which one of these mechanisms is the more important, with considerable paleoclimatic implications: If the observed increase was primarily due to more efficient long-range transport then the large increase would be limited to the remote regions; if however the increased dust flux was primarily due to increased source strength, then the increase would scale proportionally along the whole atmospheric transport pathways.

A first indication can be deduced from the ice core dust record itself: The size distribution-here given as the lognormal mode (i.e. maximum) of the volume distribution-shows larger particles during colder periods. This indicates shorter transport durations during colder periods because larger particles undergo stronger gravitational settling than small ones and are thus less depleted during times of short transport durations. Given the stationary location of the sources, a shorter transport duration implies faster transporting winds unless the pathway has changed. Quantitative estimates based on a simple 1-D transport model suggest a reduction of transport time by $\sim 25 \%$ for LGM compared to Holocene and by $\sim 10 \%$ for stadials compared to interstadials. Assuming exponential decrease of concentration during transport and a decrease of the hydrological cycle during LGM by a factor of two, this implies concentration increases by a factor of $\sim 4$ and $\sim 2$ for Holocene/LGM and interstadial/stadial, respectively (Ruth et al., 2003). Thus, only a small fraction of the observed concentration increase can be explained by enhanced transport efficiency during cold times.

Another indication can be obtained from Chinese loess archives, which are close to the source areas for Greenland ice core dust and are influenced by the same monsoonal circulation regimes. The mean diameter of loess grains is indicative of the strength of the transporting winds, which are expected to correlate with the strength of dust storms that cause the mobilisation of Greenland ice core dust. Therefore, the grain size record of the Luochuan loess section (Xiao et al., 1999) is compared to the NorthGRIP dust concentration in Figure 2. Despite its inferior time resolution, it shows remarkable similarities with the North-GRIP particle concentration. It is difficult to quantify how a change in loess granulometry should relate to the source strength for Greenland ice core dust. However, the data together with our knowledge about present-day climatology suggests a causal link between regional loess transport within China and dust mobilisation for intercontinental export of dust during the last glacial period. This notion is supported by the observed increase 


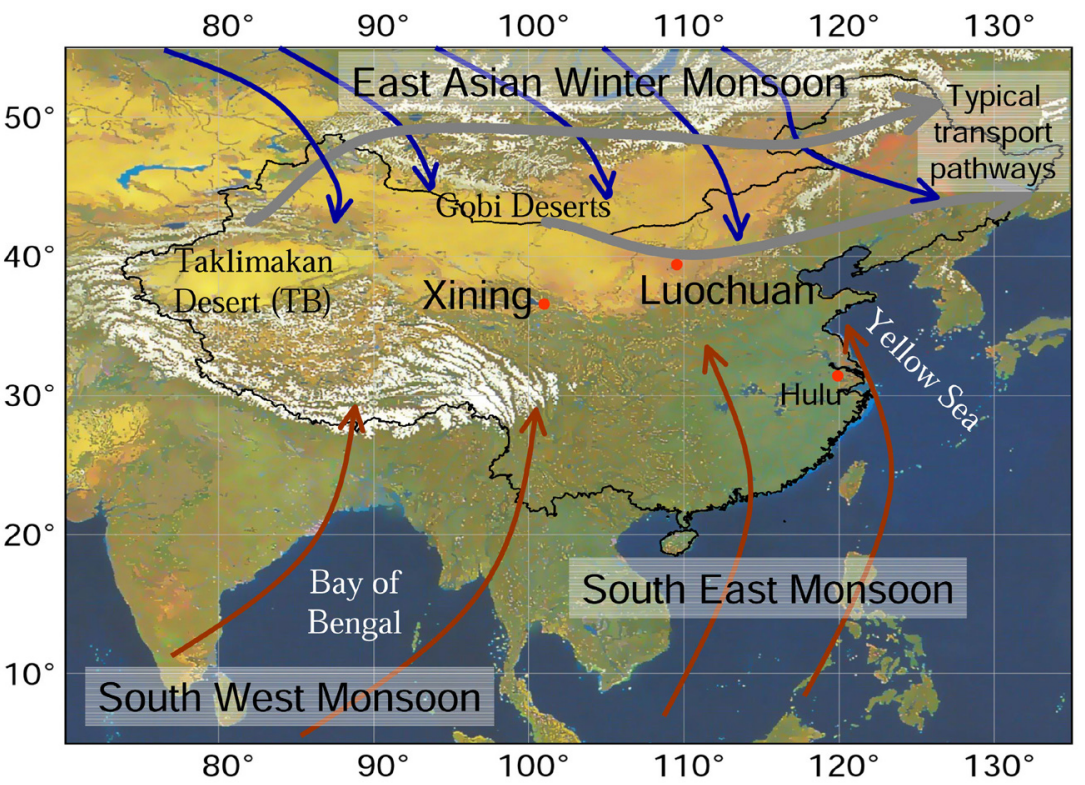

Figure 1: Map of East Asia

The Taklimakan Desert and the deserts of Inner Mongolia have been identified as the main source areas for Greenland ice core dust (TB is Tarim Basin). The loess profile discussed here is from Luochuan. Typical monsoonal circulation is illustrated along with typical transport pathways for intercontinental export of dust.

of dust flux to large regions of continental Asia and to the Pacific during the last glacial, which challenges the idea that the increased dust concentration in Greenland during that time was predominantly caused by reduced wash-out en route due to the reduced hydrological cycle.

Therefore, while bearing in mind that long-range transport also changed and did have an influence on the dust concentration in Greenland, we can interpret Greenland ice core dust concentration as a semi-quantitative proxy for occurrence and strength of dust storms in East Asia. Besides the implications for ice core interpretation, this also means that during the last glacial period the atmospheric dust load was strongly increased all along the transport pathways downwind from the sources. Thus, the proposed direct and indirect climatic impact of the dust aerosol must be considered on a hemispheric scale and not only for the high latitudes.

Further work on the composition of the dust should reveal more detailed information about possible regional changes of, and variable climate conditions in, the source areas. More high-resolution and well-dated continental climate records from East Asia are desirable to further refine the interpretation of the Greenlandic ice core dust records.

\section{References}

Bory, A., P. Biscaye, and F.E. Grousset, Two distinct seasonal Asian source regions for mineral dust deposited in Greenland (NorthGRIP), Geophysical Research Letters, 30 (4), pp1167, doi:10.1029/2002GL016446, 2003.

NGRIP-members, High-resolution record of the Northern Hemisphere climate extending into the last interglacial period, Nature, 431, 147-151, 2004.

Ruth, U., D. Wagenbach, J.P. Steffensen, and M. Bigler, Continuous record of microparticle concentration and size distribution in the central Greenland NGRIP ice core during the lastglacial period, Journal of Geophysical Research, 108 (D3), doi:10.1029/2002JD002376, 2003.

Svensson, A., P.E. Biscaye, and F.E. Grousset, Characterization of late glacial continental dust in the Greenland Ice Core Project ice core, Journal of Geophysical Research, 105 (D24), 4637-4656, 2000.

Xiao, J., Z. An, T. Liu, Y. Inouchi, H. Kumai, S. Yoshikawa, and Y. Kondo, East Asian monsoon variation during the last 130000 years: evidence from the Loess Plateau of central China and Lake Biwa of Japan, Quaternary Science Reviews, 18, 147-157, 1999.
Figure 2: Time series of ice core and synchronised loess data on the NGRIP SSO9 sea age scale Ice core $\delta 180$ (NGRIP-members, 2004), dust (insoluble particles) mass concentration and mean particle size (lognormal mode of volume distribution, diameter) (Ruth et al., 2003) together with loess grain size (quartz mean diameter, QMD) for Luochuan (Xiao et al., 1999). The loess profile has been fine-tuned to the NGRIP dust concentration profile keeping the age corrections for the loess within the reported dating uncertainties. Some stratigraphic information is given: MIS is Marine Isotope Stage, YD is Younger Dryas, B/A is Bolling/Allerod, GIS is Greenland Interstadial. Dust calibration is uncertain for the peak marked "?" at $17.0 \mathrm{ka}$.

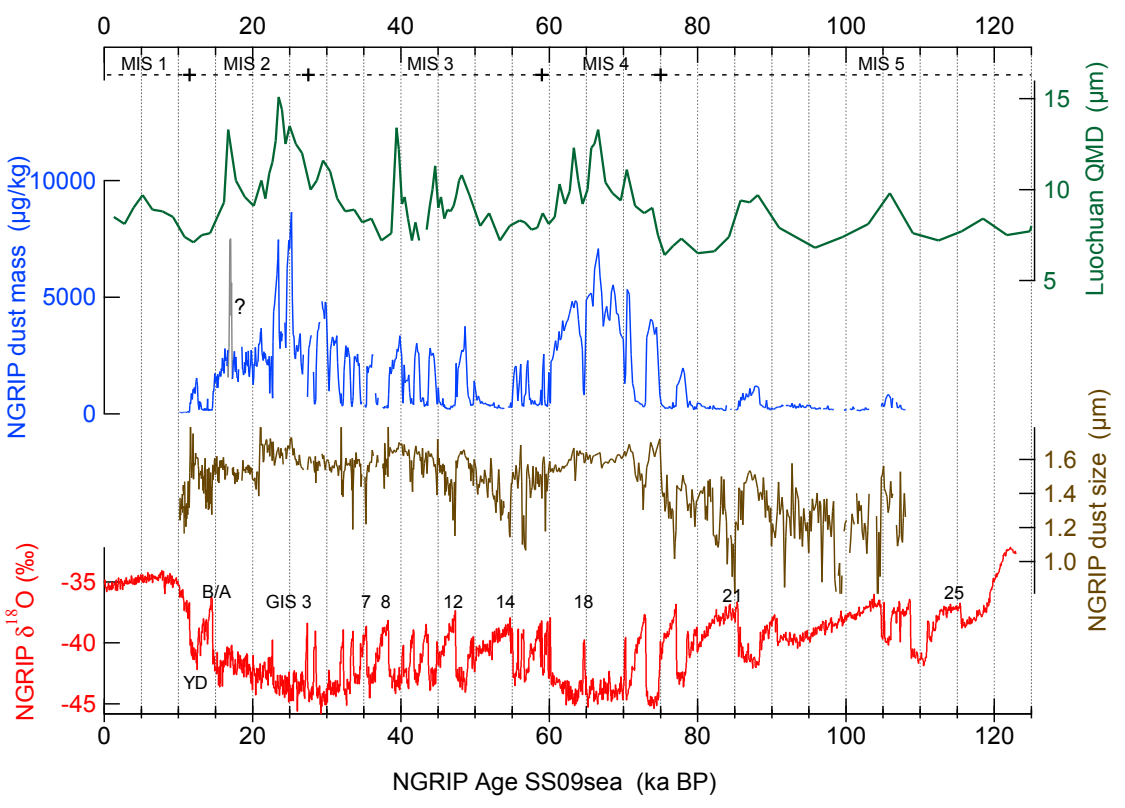

18 